\title{
A serum metabolomic investigation on lipoprotein lipase-deficient mice with hyperlipidemic pancreatitis using gas chromatography/mass spectrometry
}

\author{
MAOCHUN TANG ${ }^{1 *}$, GOUYONG HU $^{1 *}$, YAN ZHAO $^{1}$, MINGMING SU $^{2}$, YUHUI WANG $^{3}$, \\ WEI JIA ${ }^{2}$, YUNPING QIU ${ }^{4}$, GEORGE LIU $^{3}$ and XINGPENG WANG ${ }^{1}$
}

${ }^{1}$ Department of Gastroenterology, Shanghai Tenth People's Hospital, Tongji University School of Medicine, Shanghai 200072;
${ }^{2}$ School of Pharmacy, Shanghai Jiao Tong University, Shanghai 200240; ${ }^{3}$ Institute of Cardiovascular Sciences and
Key Laboratory of Molecular Cardiovascular Sciences, Ministry of Education, Peking University, Beijing 100083;
${ }^{4}$ Shanghai Institute for Systems Biomedicine, Shanghai Jiao Tong University, Shanghai 200240, P.R. China

Received January 16, 2013; Accepted March 6, 2013

DOI: $10.3892 /$ br.2013.78

\begin{abstract}
Hypertriglyceridemia (HTG) is often associated with acute pancreatitis. The relationship between these diseases and the role that hypertriglyceridemia plays in acute pancreatitis pathogenesis remains to be elucidated. In the present study, in order to investigate the mechanisms of hyperlipidemic acute pancreatitis (HLP), we established an animal model using lipoprotein lipase (LPL)-deficient heterozygous mice injected with caerulein. Susceptibility to pancreatitis in LPL-deficient heterozygous mice was compared with that of wild-type mice after intraperitoneal (i.p.) injections of caerulein by determining amylase release and pancreatic pathological scores. Furthermore, serum metabolome was detected through chemical derivatization followed by gas chromatography/mass spectrometry (GC/MS). GC/MS data were analyzed by orthogonal-projection to latent structures-discriminant analysis (OPLS-DA). Caerulein induced increased levels of serum amylase and more severe pancreatic inflammation in LPL-deficient mice compared to wild-type
\end{abstract}

Correspondence to: Professor Xingpeng Wang or Dr Yan Zhao, Department of Gastroenterology, Shanghai Tenth People's Hospital, Tongji University School of Medicine, 301 Yanchang Middle Road, Shanghai 200072, P.R. China

E-mail: wangxingpeng1965@yahoo.cn

E-mail: 320zhaoyan@163.com

*Contributed equally

Abbreviations: FFA, free fatty acid; HTG, hypertriglyceridemia; GC/MS, gas chromatography/mass spectrometry; PCA, principal component analysis; OPLS-DA, orthogonal projections to latent structures discriminant analysis; TG, triglyceride; HLP, hyperlipidemic acute pancreatitis; H\&E, hematoxylin and eosin stain; LPL, lipoprotein lipase

Key words: hyperlipidemic acute pancreatitis, metabolism, blood, lipoprotein lipase mice. HLP was discriminated more accurately from healthy controls by the metabolites, including valine, leucine and citrate. The metabolites included valine, leucine, citrate, malic acid, proline, tetradecanoic acid (14:0), glutamine and oleic acid (18:1). Changes in energy, fat and amino acid metabolism were also evident. In conclusion, LPL-deficient heterozygous mice with hypertriglyceridemia (HTG) exhibited enhanced susceptibility to acute pancreatitis. GC/MS data revealed differences between healthy and HLP mice. Therefore, this technique is novel and a useful tool for the study of the HLP pathogenetic mechanism.

\section{Introduction}

Hypertriglyceridemia (HTG) is associated with acute pancreatitis in $12-38 \%$ of reported cases. The relationship between these diseases and the role of hypertriglyceridemia in acute pancreatitis pathogenesis remains to be elucidated (1). Although the pathogenesis of acute pancreatitis depends on a number of factors, its recurrent form is the most common complication of HTG and is often observed in type I hyperlipoproteinemic patients with various lipoprotein lipase (LPL) or apolipoprotein CII gene mutations (2). LPL-deficient HTG heterozygous mice were rescued by somatic gene transfer of a mutant LPL gene. The surviving animals exhibited LPL deficiency with high plasma triglyceride (TG) levels during adulthood (3). Thus, this study aimed to assess the susceptibility of LPL-deficient heterozygous mice with HTG to pancreatitis. Furthermore, gas chromatography/mass spectrometry (GC/MS) was utilized to compare serum metabolite levels between normal controls and hyperlipidemic acute pancreatitis (HLP) subjects.

The recent revival of systems biology that integrates the pertinent components, such as genes, proteins and metabolites, into a holistic biological network provides a comprehensive understanding of system behavior $(4,5)$. Metabolomic profiling is a practical approach used to determine system response to perturbations by measuring variations in small molecule/ endpoint metabolic products resulting from systemic biochemical regulation (6). In this study, we aimed to demonstrate the 
use of GC/MS for HLP serum metabolic analysis, and provide potential metabolic biomarkers to distinguish HLP subjects from normal subjects.

\section{Materials and methods}

Animals. LPL-deficient mice were rescued from neonatal death by intramuscular injection of an adenoviral vector coding a naturally occurring human LPL beneficial mutant, Ad-LPLS447X, as previously described (3). Animals injected with saline were used as the control. Genotyping was performed by polymerase chain reaction. LPL-deficient heterozygous mice were hybridized by female wild-type and LPL-deficient mice. Female wild-type and LPL-deficient mice with a C57B6 background at 8 weeks weighing 13-16 g were used for the experiments. The animals were fed a normal chow diet and had free access to water. The 'Principles of Laboratory Animal Care' (NIH publication no. 85-23, revised 1996) was followed and the experimental protocol was approved by the Animal Care Committee, Peking University Health Science Center.

Experimental group. The experimental protocol is shown in Fig. 1. Four groups were prepared: group 1 [WTC, wild-type control: $0.9 \%$ sodium chloride was intraperitoneally (i.p.) injected 7 times over 1 day], group 2 (LDC, LPL-deficient heterozygous control), group 3 [WTT, acute pancreatitis model; caerulein was i.p. injected 7 times over 1 day, Sigma Aldrich Chemie GmbH (Steinheim, Germany) dosage, $20 \mu \mathrm{g} / \mathrm{kg}$ b.w. every hour] and group 4 (LDT HLP model). Blood samples were collected $12 \mathrm{~h}$ after injection to measure amylase, triacylglycerol (TG) and conduct GC/MS analysis. Mice were then sacrificed and pancreatic tissues were fixed in buffered formalin and embedded in paraffin.

Biochemical measurement and histopathology. Serum was analyzed for amylase and TG concentrations using commercial kits (Biosino Bio-Technology and Science, Beijing, China). Histological samples were fixed in $10 \%$ buffered formalin, embedded in paraffin, sectioned and stained with hematoxylin and eosin stain $(\mathrm{H} \& \mathrm{E})$.

GC/MS sample preparation, derivatization and spectral acquisition. Serum samples collected from fasting subjects were kept frozen at $-80^{\circ} \mathrm{C}$ until use, at which point the ice samples were thawed. Each 200- $\mu 1$ aliquot of the serum samples was added to a $1.5 \mathrm{ml}$ tube followed by the addition of $400 \mu 1$ of acetone for protein precipitation. The mixture was vortexed for $30 \mathrm{sec}$ and centrifuged at $10,000 \mathrm{x}$ g for $10 \mathrm{~min}$. A $400-\mu 1$ supernatant was transferred to a $500 \mu$ l of glass tube and dried under vacuum. The dried analytes were dissolved in $80 \mu \mathrm{l}$ of methoxylamine hydrochloride $(15 \mathrm{mg} / \mathrm{ml}$, dissolved in pyridine) for $90 \mathrm{~min}$ at $30^{\circ} \mathrm{C}$ and then silylated with $80 \mu \mathrm{l}$ N,O-bis(trimethylsilyl)-trifluoroacetamide (BSTFA) and trimethylchlorosilane (at a ratio of 99:1) (Supelco, Bellefonte, PA, USA) for $2 \mathrm{~h}$ at $70^{\circ} \mathrm{C}$. Each $70-\mu \mathrm{l}$ aliquot of hexane was added to the derivatization flasks. After the sample was stirred for $1 \mathrm{~min}$ and kept at room temperature for $1 \mathrm{~h}, 1-\mu 1$ aliquot of the solution was injected into a PerkinElmer GC coupled with a TurboMass-Autosystem XL mass spectrometer (PerkinElmer,

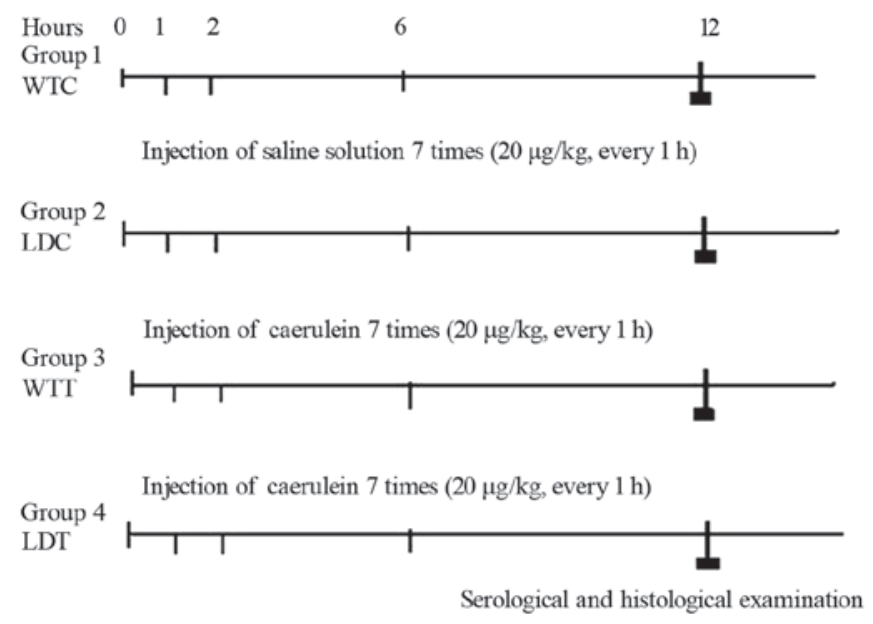

Figure 1. Experimental protocol. Mice in groups 1 and 2 mice were treated with $0.9 \%$ sodium chloride by i.p. injection. In groups 3 and 4, caerulein was i.p. injected 7 times (dosage, $20 \mu \mathrm{g} / \mathrm{kg}$ every h) over 1 day. Bleeding and histopathologic examinations were performed $12 \mathrm{~h}$ after injection.

Inc., St. Waltham, MA, USA) in the splitless mode. A DB-5MS capillary column coated with 5\% diphenyl cross-linked $95 \%$ dimethylpolysiloxane (30 m x $250 \mu \mathrm{m}$ i.d., $0.25-\mu \mathrm{m}$ film thickness; Agilent J\&W Scientific, Folsom, CA, USA) was used for separation. The injection and interface temperatures were set to $260^{\circ} \mathrm{C}$ and the ion source temperature was adjusted to $200^{\circ} \mathrm{C}$. Initial GC oven temperature was set at $80^{\circ} \mathrm{C}$ for $2 \mathrm{~min}$ following injection and then raised up to $285^{\circ} \mathrm{C}$ with $5^{\circ} \mathrm{C} / \mathrm{min}$ and maintained at $285^{\circ} \mathrm{C}$ for $7 \mathrm{~min}$. Helium at a flow rate of $1 \mathrm{ml} / \mathrm{min}$ was used as the carrier gas. Measurements were made with electron impact ionization $(70 \mathrm{eV})$ in the full scan mode (m/z 30-550) (7).

Data analysis. Biochemical measurement results were expressed as the mean \pm standard deviation (SD). Statistical evaluation was performed with SPSS 11.0 and differences between the 2 groups were tested by independent t-tests. $\mathrm{P}<0.05$ was considered statistically significant.

GC/MS data files were converted into NetCDF format via DataBridge (PerkinElmer, Inc.) and pretreatment was conducted as previously described (8). The mean-centered and autoscaled data were then introduced into SIMCA-P 11.5 Software (Umetrics, Umeå, Sweden) for multivariate statistical analysis. Principal component analysis (PCA) was used to obtain an overview of variations among the different groups. Orthogonal-projections to latent structures-discriminant analysis (OPLS-DA), a supervised pattern recognition approach, was utilized to construct a predictive model to identify the differential metabolites involved in causing disease. To avoid the overfitting of the models, the OPLS-DA model was carefully validated by the following three steps: i) an iterative 7-round cross-validation with one seventh of the samples being excluded from the model in each round (9); ii) 1,000 random permutations test (10); iii) blind prediction test in which the data set was randomly divided into the training set (70\%) and test set (30\%) and the model built on the training set was applied to construct the classification model to predict the class membership of the test set (11). 
Table I. Plasma triglyceride (TG) and plasma amylase levels in pancreatic injury.

Wild-type control LPL-deficient control Wild-type, after caerulein LPL-deficient, after caerulein

\section{Plasma TG}

$\mathrm{mm} / \mathrm{l}(\mathrm{n}=7)$

$$
0.91 \pm 0.02
$$

$3.58 \pm 0.03$

$0.93 \pm 0.03$

$3.56 \pm 0.02$

Plasma amylase

$\mu / 1(\mathrm{n}=7)$

$358.33 \pm 42.72$

$471.29 \pm 22.74$

$2500.89 \pm 409.59^{\mathrm{a}}$

$3685.06 \pm 483.98^{\mathrm{a}}$

${ }^{\mathrm{a}} \mathrm{P}<0.05$. Correlation coefficients are based on orthogonal-projections to latent structures-discriminate analysis (OPLS-DA) analysis of the two-group model (group 1 vs. 4); positive values indicate metabolite increase in group 4. LPL, lipoprotein lipase.

A

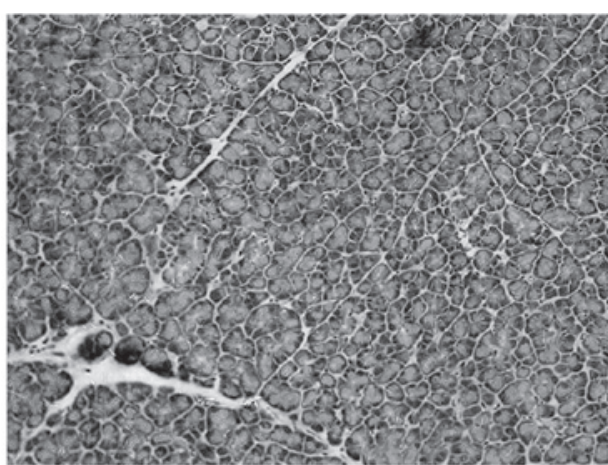

C

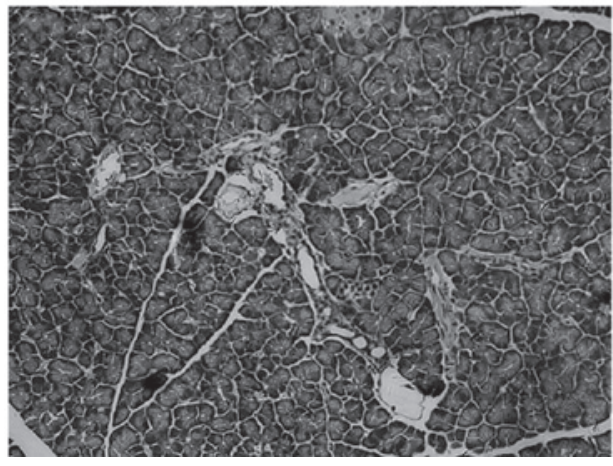

B

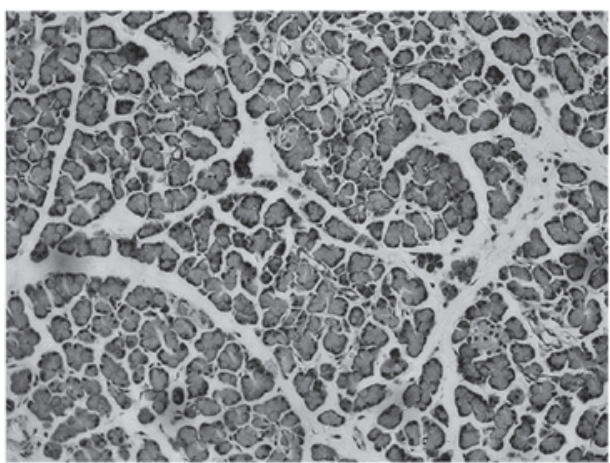

D

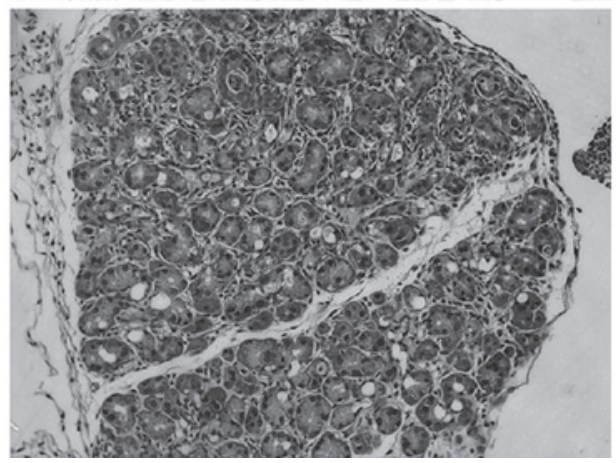

Figure 2. Hematoxylin and eosin (H\&E)-stained sections of pancreatic tissue (magnification, x400). (A and B) Groups 1 and 3 i.p.-injected wild-type control and caerulein-treated mice, (C and D) groups 2 and 4 lipoprotein lipase (LPL)-deficient heterozygous control and caerulein-treated mice (7 i.p. injections, $20 \mu \mathrm{g} / \mathrm{kg}$ b.w. at hourly intervals); (D) showed more severe edema, inflammation, hemorrhage and necrosis.

\section{Results}

Histopathologic observation. No discernable pathological alteration in either wild-type or LPL-deficient heterozygous mice injected relative to the saline-injected control was observed. However, mice injected with caerulein exhibited pancreatic injury including edema, neutrophil infiltration, hemorrhage and parenchymal necrosis. In LPL-deficient heterozygous mice injected with caerulein, pancreatic injury was more severe compared to other mice (Fig. 2).

Measurement of serum amylase and plasma triglyceride (TG) levels. Serum amylase levels increased after caerulein injection in both wild-type and LPL-deficient mice, but were significantly higher in LPL-deficient heterozygous mice $12 \mathrm{~h}$ after injection. The difference between wild-type control and LPL-deficient control mice was not significant. Moreover, the plasma TG levels in LPL-deficient mice were significantly higher than the control animals (Table I, $\mathrm{P}<0.05$ ).
GC/MS spectroscopic data analysis. Compound identification was carried out either by comparing mass spectra and retention time with values obtained from commercially available reference compounds or based on commercial libraries of NIST, NBS and Wiley. We were able to identify metabolites, primarily organic acids, amino acids and free fatty acids (FFA). The OPLS-DA model calculated from GC/MS data of HLP vs. wild-type subjects was employed. There were more differentially expressed metabolites identified in HLP compared to the healthy group (Table II and Fig. 3), including compounds such as valine, leucine, glutamine, proline, citrate, malic acid, tetradecanoic acid (14:0) and oleic acid (18:1).

\section{Discussion}

HLP typically presents as an episode of acute pancreatitis or recurrent acute pancreatitis (1) and has a high morbidity rate. It is important to determine the pathogenetic factors involved as the disease is treatable and recurrences can be prevented (12). 
Table II. Statistical analysis of differentially expressed endogenous metabolites correlated with HLP and wild-type mice.

\begin{tabular}{|c|c|c|c|}
\hline $\mathrm{Rt} / \mathrm{min}$ & Metabolites & Correlation coefficient & KEGG pathway \\
\hline 9.38 & Valine & 0.32 & Amino acid metabolism \\
\hline 10.42 & Leucine & 0.33 & Amino acid metabolism \\
\hline 15.21 & Citrate & -0.26 & Energy metabolism \\
\hline 16.24 & Malic acid & -0.3 & Energy metabolism \\
\hline 17.00 & Proline & 0.3 & Amino acid metabolism \\
\hline 17.34 & Tetradecanoic acid (14:0) & 0.26 & Fatty acid metabolism \\
\hline 17.36 & Glutamine & -0.35 & Energy metabolism \\
\hline 31.32 & Oleic acid (18:1) & 0.32 & Fatty acid metabolism \\
\hline
\end{tabular}

HLP, hyperlipidemic acute pancreatitis; RT, retention time; KEGG, Kyoto encyclopedia of genes and genomes.

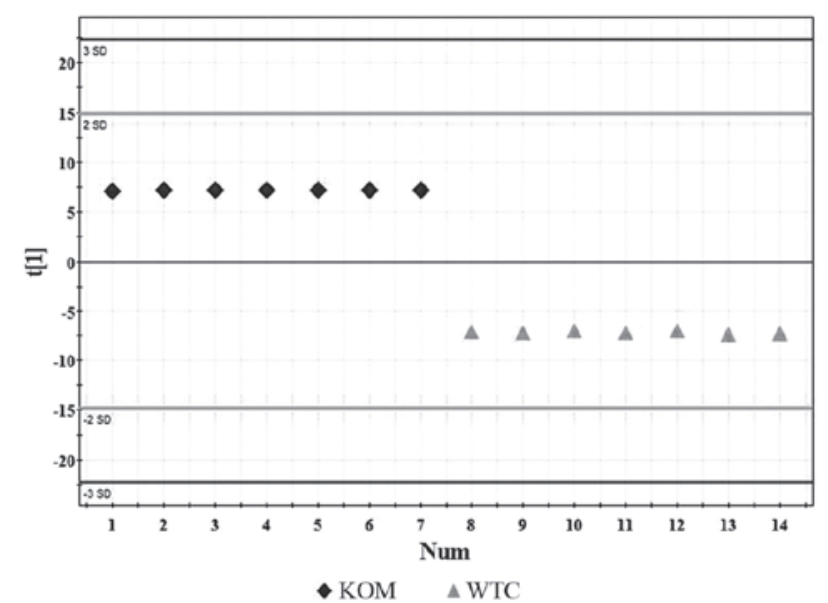

Figure 3. Serum orthogonal-projections to latent structures-discriminant analysis (OPLS-DA) of lipoprotein lipase (LPL)-deficient heterozygous experimental mice (group 4) and wild-type mice (group 1).

The mechanisms by which hyperlipidemia induces acute severe pancreatitis have yet to be elucidated.

LPL-deficient heterozygous mice developed pancreatitis subequent to caerulein stimulation. It appears that pancreatic cells are more susceptible to damage by an additional stimulus or injury in the presence of high TG plasma levels surrounding pancreatic cells (13). Once cells are damaged, high concentrations of pancreatic lipase, exceeding normal blood plasma levels, may reach the interstitium.

Although various animal models for chronic, acute or severe pancreatitis have previously been established and investigated (14-16), no appropriate HLP model has been developed. In this study, by using the combination of LPL-deficient heterozygous mice and injection with caerulein, we established animal models for HLP and conducted further studies using these models.

Since HLP is a metabolic disorder that disturbs the metabolism of carbohydrates, lipids and amino acids, the pathological process likely involves an altered expression of downstream low molecular weight metabolites such as glucose. This study was designed to visualize the alteration of global serum metabolites associated with HLP pathophysiology. Compared to healthy controls, the HLP subjects exhibited altered serum metabolites (Table II) including significantly increased valine, leucine, proline, tetradecanoic acid (14:0) and oleic acid (18:1), as well as decreased citrate, malic acid and glutamine levels. Several pathways involving the tricarboxylic acid (TCA) cycle (citrate and malic acid) and glutamate and glutamine biosynthesis were affected.

Citrate, malic acid and glutamine are crucial components of the TCA cycle, which is the main pathway of glucose degradation and primary energy supplier for most organisms. These compounds were significantly decreased in the HLP subjects than in the healthy control animals, suggesting that HLP may inhibit the activity of these compounds, ultimately repressing energy metabolism. As the metabolic enzymes of the TCA cycle are located mainly in the mitochondria, TCA cycle disorder results in disrupted mitochondrial function.

Compared to healthy controls, the HLP subjects exhibited altered serum metabolites (Table II) including significantly increased oleic acid (18:1) and tetradecanoic acid (14:0). This suggests a hypercatabolic state in HLP subjects, which is consistent with previously reported results (17-19). A well-accepted mechanism initially proposed by Havel (20) states that TG hydrolysis in and around the pancreas by pancreatic lipase seeping out of the acinar cells, leading to the accumulation of FFA in high concentrations. FFA may disturb microcirculation in the pancreas via detrimental effects on the vessel endothelium $(21,22)$. A metabolic factor may play a key role in HLP pathogenesis.

In conclusion, LPL-deficient mice with high HTG have enhanced susceptibility to pancreatitis. Serum metabolite profiling using $\mathrm{GC} / \mathrm{MS}$ in conjunction with modern multivariate statistical techniques permits non-invasive, simultaneous monitoring of numerous metabolic pathways. Significantly altered serum metabolites were detected in HLP subjects and these changes impact some biochemical pathways.

\section{Acknowledgements}

This study was supported by the National Natural Science Foundation of China (grant no. 30971359). We would like to thank the technical support of Li Cheng, Zhenjun Gao and Kai Wu at the Department of Gastroenterology of Shanghai Jiaotong University Affiliated First People's Hospital. 


\section{References}

1. Yadav D and Pitchumoni CS: Issues in hyperlipidemic pancreatitis. J Clin Gastroenterol 36: 54-62, 2003.

2. Toskes PP: Hyperlipidemic pancreatitis. Gastroenterol Clin North Am 19: 783-791, 1990.

3. Ross CJ, Liu G, Kuivenhoven JA, et al: Complete rescue of lipoprotein lipase-deficient mice by somatic gene transfer of the naturally occurring LPLS447X beneficial mutation. Arterioscler Thromb Vasc Biol 25: 2143-2150, 2005.

4. Hood L and Perlmutter RM: The impact of systems approaches on biological problems in drug discovery. Nat Biotechnol 22 1215-1217, 2004.

5. Hwang D, Rust AG, Ramsey S, et al: A data integration methodology for systems biology. Proc Natl Acad Sci USA 102 17296-17301, 2005.

6. Nicholson JK, Holmes E and Wilson ID: Gut microorganisms, mammalian metabolism and personalized health care. Nat Rev Microbiol 3: 431-438, 2005.

7. Bao Y, Zhao T, Wang X, et al: Metabonomic variations in the drug-treated type 2 diabetes mellitus patients and healthy volunteers. J Proteome Res 8: 1623-1630, 2009.

8. Wang X, Su M, Qiu Y, et al: Metabolic regulatory network alterations in response to acute cold stress and ginsenoside intervention. J Proteome Res 6: 3449-3455, 2007.

9. Ni Y, Su M, Lin J, et al: Metabolic profiling reveals disorder of amino acid metabolism in four brain regions from a rat model of chronic unpredictable mild stress. FEBS Lett 582: 2627-2636, 2008.

10. Westerhuis JA, Hoefsloot HCJ, Smit S, et al: Assessment of PLSDA cross validation. Metabolomics 4: 81-89, 2008.

11. Jellum E, Bjornson I, Nesbakken R, et al: Classification of human cancer cells by means of capillary gas chromatography and pattern recognition analysis. J Chromatogr 217: 231-237, 1981.
12. Karne S and Gorelick FS: Etiopathogenesis of acute pancreatitis Surg Clin North Am 79: 699-710, 1999.

13. Wang Y, Sternfeld L, Yang F, et al: Enhanced susceptibility to pancreatitis in severe hypertriglyceridaemic lipoprotein lipase-deficient mice and agonist-like function of pancreatic lipase in pancreatic cells. Gut 58: 422-430, 2009.

14. Frossard JL and Pastor CM: Experimental acute pancreatitis: new insights into the pathophysiology. Front Biosci 7: d275-d287, 2002.

15. Esposito I, Friess H and Buchler MW: Molecular mechanisms in chronic pancreatitis. Zentralbl Chir 126: 867-872, 2001.

16. Gaeta L, Dionigi G and Dionigi R: Experimental models of acute pancreatitis. Critical review. Chir Ital 52: 469-497, 2000 (In Italian).

17. Chen M, Zhao L and Jia W: Metabonomic study on the biochemical profiles of a hydrocortisone-induced animal mode. J Proteome Res 4: 2391-2396, 2005.

18. Chen M, Su M, Zhao L, et al: Metabonomic study of aristolochic acid-induced nephrotoxicity in rats. J Proteome Res 5: 995-1002, 2006.

19. Wang Y, Holmes E, Nicholson JK, et al: Metabonomic investigations in mice infected with Schistosoma mansoni: an approach for biomarker identification. Proc Natl Acad Sci USA 101: 12676-12681, 2004.

20. Havel RJ: Pathogenesis, differentiation and management of hypertriglyceridemia. Adv Intern Med 15: 117-154, 1969.

21. Hofbauer B, Saluja AK, Lerch MM, et al: Intra-acinar cell activation of trypsinogen during caerulein-induced pancreatitis in rats. Am J Physiol 275: G352-G362, 1998.

22. Mayer J, Rau B, Schoenberg MH, et al: Mechanism and role of trypsinogen activation in acute pancreatitis. Hepatogastroenterology 46: 2757-2763, 1999. 